\title{
The Kv2.I $\mathrm{K}^{+}$channel targets to the axon initial segment of hippocampal and cortical neurons in culture and in situ Patrick D Sarmiere ${ }^{1,2}$, Cecile M Weigle ${ }^{1}$ and Michael M Tamkun*1
}

Address: ${ }^{1}$ Program in Molecular, Cellular, and Developmental Neuroscience, Department of Biomedical Sciences \& Department of Biochemistry and Molecular Biology, Colorado State University, Fort Collins, Colorado, USA and ${ }^{2}$ Spinal Cord Society Research Center, 2401 Research Blvd. Suite 206, Fort Collins, Colorado, USA

Email: Patrick D Sarmiere - psarmiere@scs.cbeyond.com; Cecile M Weigle - cecile.weigle@ colostate.edu; Michael M Tamkun* - tamkunmm@lamar.colostate.edu

* Corresponding author

Published: 13 November 2008

BMC Neuroscience 2008, 9:1 I2 doi:10.1 |86/147|-2202-9-1 /2
Received: 14 July 2008

Accepted: 13 November 2008

This article is available from: http://www.biomedcentral.com/I47/-2202/9/II2

(C) 2008 Sarmiere et al; licensee BioMed Central Ltd.

This is an Open Access article distributed under the terms of the Creative Commons Attribution License (http://creativecommons.org/licenses/by/2.0), which permits unrestricted use, distribution, and reproduction in any medium, provided the original work is properly cited.

\begin{abstract}
Background: The Kv2.I delayed-rectifier $\mathrm{K}^{+}$channel regulates membrane excitability in hippocampal neurons where it targets to dynamic cell surface clusters on the soma and proximal dendrites. In the past, Kv2.I has been assumed to be absent from the axon initial segment.

Results: Transfected and endogenous $\mathrm{Kv} 2 . \mathrm{I}$ is now demonstrated to preferentially accumulate within the axon initial segment (AIS) over other neurite processes; $87 \%$ of I4 DIV hippocampal neurons show endogenous channel concentrated at the AIS relative to the soma and proximal dendrites. In contrast to the localization observed in pyramidal cells, GAD positive inhibitory neurons within the hippocampal cultures did not show AIS targeting. Photoactivable-GFP-Kv2.Icontaining clusters at the AIS were stable, moving $<1 \mu \mathrm{m} / \mathrm{hr}$ with no channel turnover. Photobleach studies indicated individual channels within the cluster perimeter were highly mobile (FRAP $\tau=$ $10.4 \pm 4.8 \mathrm{sec}$ ), supporting our model that $\mathrm{Kv} 2$.I clusters are formed by the retention of mobile channels behind a diffusion-limiting perimeter. Demonstrating that the AIS targeting is not a tissue culture artifact, $\mathrm{Kv} 2 . \mathrm{I}$ was found in axon initial segments within both the adult rat hippocampal CAI, CA2, and CA3 layers and cortex.

Conclusion: In summary, Kv2.I is associated with the axon initial segment both in vitro and in vivo where it may modulate action potential frequency and back propagation. Since transfected Kv2.I initially localizes to the AIS before appearing on the soma, it is likely multiple mechanisms regulate $\mathrm{Kv2.I}$ trafficking to the cell surface.
\end{abstract}

\section{Background}

Voltage-gated ion channels are often highly localized in electrically excitable cells such as nerve and muscle. As originally noted by Trimmer and colleagues [1], the Kv2.1 delayed rectifier is expressed primarily in the somatic region of hippocampal neurons where it is found in cell surface clusters that can co-localize with ryanodine recep- tors and SR-like subsurface cisterns [2,3]. Interestingly, these clusters also co-localize with cholinergic synapses in spinal motor neurons [4]. Kv2.1 represents the predominant delayed rectifier current in hippocampal neurons where its activity and localization are highly regulated $[5,6]$. Glutamate or carbachol treatments induce both Kv2.1 dephosphorylation and declustering [7-9]. Both 
treatments also result in a $20 \mathrm{mV}$ hyperpolarizing shift in the activation curve for $\mathrm{I}_{\mathrm{K}}$. Chemically-induced ischemia also induces declustering, dephosphorylation, and the hyperpolarizing shift in the activation midpoint $[8,9]$. Similar regulation is observed in Kv2.1 transfected HEK cells [9]. These data suggest a strong link between cluster formation, channel phosphorylation, and the voltagedependence of activation. The increase in channel activity that is linked to declustering has been proposed to be a neuro-protective response to hypoxia/ischemic insult [10]. However, Kv2.1 trafficking to the cell surface is also implicated in cortical neuron apoptosis $[11,12]$, emphasizing that the trafficking and regulation of Kv2.1 must be under tight physiological control.

While it is commonly assumed that ion channel localization must involve static tethering to scaffolding proteins that in turn are linked directly to the cytoskeleton, our recent studies indicate that the Kv2.1 surface clusters are formed when mobile Kv2.1 channels are corralled behind a cortical actin-based fence [13]. This sub-membrane fence is selective towards only the confined channels, with other membrane proteins being free to cross it. Thus, the Kv2.1-containing surface clusters represent a new mechanism for the stable localization of ion channel proteins to specific cell surface domains. Our previous studies also indicate that the surface clusters are specialized surface sites for the membrane insertion of Kv2.1 channels, functioning as intracellular trafficking vesicle targets [14]. During the course of our studies we often observed GFP-Kv2.1 clusters forming in a single proximal neurite of a transfected hippocampal neuron. While the expression of Kv2.1 within the axon initial segment (AIS) of cultured hippocampal neurons has previously been referred to as a tissue culture artifact [8], AIS localization was often the only cell surface expression observed in an individual cell. The study presented here was initiated by this apparent contradiction between the literature and our data obtained in hippocampal neurons transfected with GFPKv2.1.

We report here that both transfected and endogenous Kv2.1 often show a real preference for the AIS in cultured hippocampal neurons. The Kv2.1 clusters within the AIS are similar to those found on the cell body in that they consist of mobile channels trapped by a perimeter fence. However, perhaps due to the sub-membrane diffusion barriers in the AIS [15-17], the clusters themselves appear to be more confined than their cell body counterparts [14]. Kv2.1 concentration within the AIS also occurs in both cortical and hippocampal neurons of adult brain, confirming that AIS localization is not a tissue culture artifact. AIS-localized Kv2.1 is predicted to regulate both the frequency and back propagation of the axonal action potential.

\section{Results \\ Exogenously expressed Kv2. I targets to the axon initial segment in cultured hippocampal neurons before accumulating on the soma}

Our initial imaging experiments involved expressing GFP and HA epitope-tagged Kv2.1 (EGFP-Kv2.1-HA) in rat hippocampal neurons grown in culture for 7-9 days. The extracellular HA epitope allowed detection of surface channel via the binding of an anti-HA Alexa-594-conjugated antibody to living cells as previously described [14]. In contrast to the expected soma and proximal neurite expression [7,18], 85\% of neurons expressing EGFPKv2.1-HA exhibited surface accumulation at only one of the proximal neurites at $6 \mathrm{~h}$ post transfection (see arrows in Fig. 1, 6 h). This targeting of Kv2.1 to a single proximal neurite was observed with little or no clustering on the cell body. In neurons expressing Kv2.1 for 18 h, surface clusters within the soma (arrowheads) became more apparent relative to that observed at $6 \mathrm{~h}$ postransfection (Fig. 1, 18 h). By $24 \mathrm{~h}$ after transfection, the classic distribution of Kv2.1 into compact surface clusters was observed on the cell body along with expression in proximal neurites. However, Kv2.1 surface density remained the greatest in a single proximal neurite (Fig. 1, $24 \mathrm{~h}$ ). Note that the majority of the cell body GFP fluorescence at the $6 \mathrm{~h}$ and $18 \mathrm{~h}$ time points most likely represents ER-localized channel. These representative data, showing that expressed Kv2.1 is targeted to a single proximal neurite prior to clustering at other surface somato-dendritic sites, suggest that Kv2.1 may have a preference for the axon initial segment (AIS) and may be targeted to this region via a mechanism distinct from the one responsible for delivery to somatic sites.

A defining characteristic of the AIS in hippocampal neurons is the enrichment of ankyrinG (AnkG) in the first 20$40 \mu \mathrm{m}$ of the unmyelinated axon $[19,20]$. In addition, MAP2 immuno-reactivity is greatly reduced within the axon but is maintained within dendrites and the cell body $[7,18]$. Thus, MAP2 and AnkG expression were used to define the AIS in our culture system. Neurons transfected with EGFP-Kv2.1-HA were fixed approximately $18 \mathrm{~h}$ after transfection and immuno-stained for MAP2 or AnkG as illustrated in Fig. 2. The clustering of Kv2.1 in the proximal segment of a neurite with reduced MAP2 labeling suggests this neurite is likely to develop into the axon and therefore the proximal AIS (Fig. 2A). However, a stronger indication that the region of Kv2.1 accumulation is indeed the AIS is the AnkG staining shown in Fig. $2 \mathrm{~B}$. Consistent with AIS formation in hippocampal cultures, Nav1.2 co-localized with AnkG and was enriched in areas devoid of MAP2 staining in parallel cultures (data not shown). 

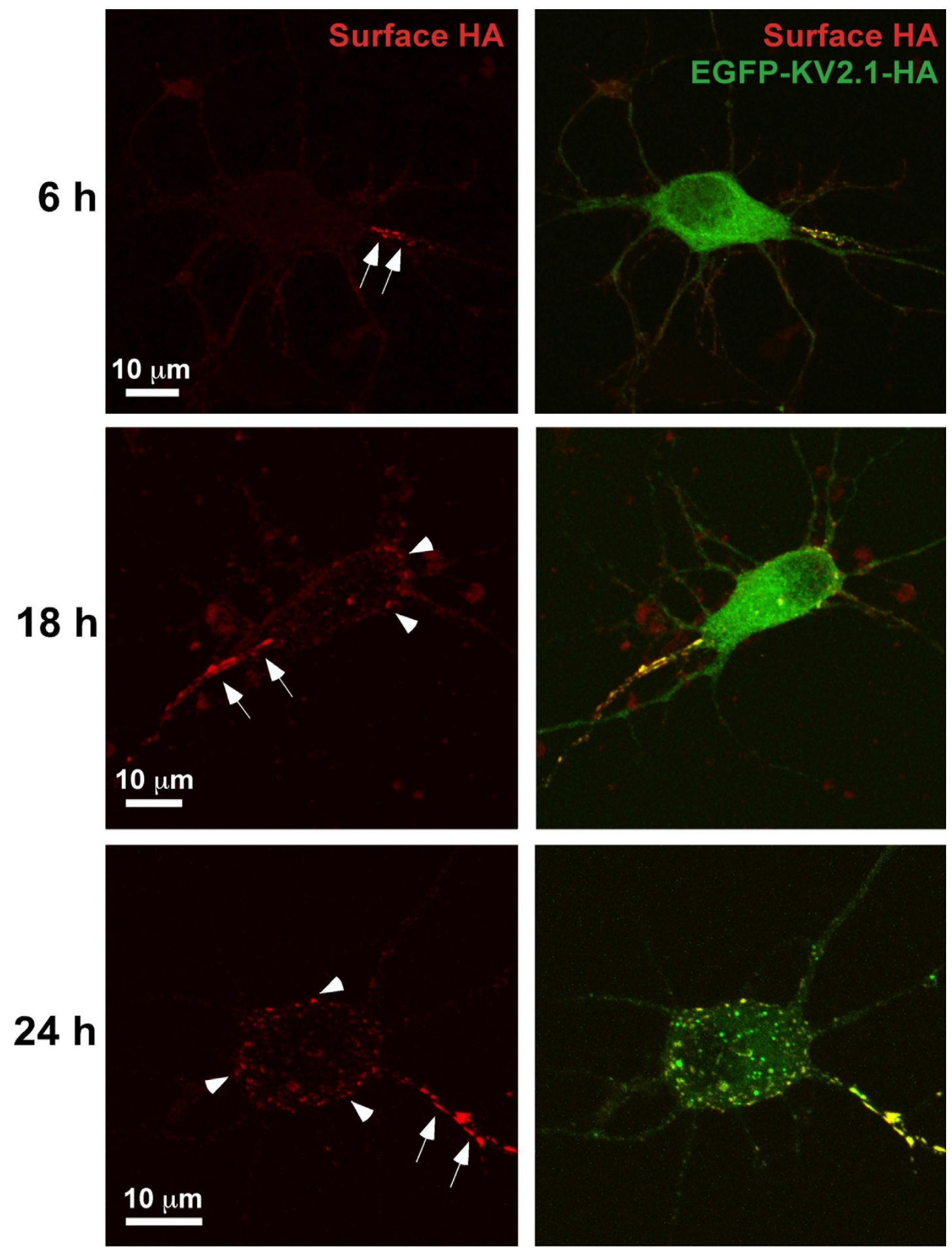

Figure I

Surface accumulation of transfected Kv2.I in a single neurite. Hippocampal neurons transfected with EGFP-Kv2.I-HA for 6, 18 and $24 \mathrm{~h}$ were labeled with Alexa 594-conjugated anti-HA monoclonal antibody 30 min prior to live cell imaging. Shown are representative maximum projection images comprised of multiple $0.3 \mu \mathrm{m}$ optical sections for total EGFP-Kv2.I-HA (green, GFP signal) and surface (red, Alexa 594 anti-HA antibody binding). Kv2.I surface clusters were observed exclusively in single a proximal neurite as early as 6 hours after transfection (arrows). At 18 and 24 hours post-transfection, the appearance of surface somato-dendritic clustering became apparent (arrowheads). 

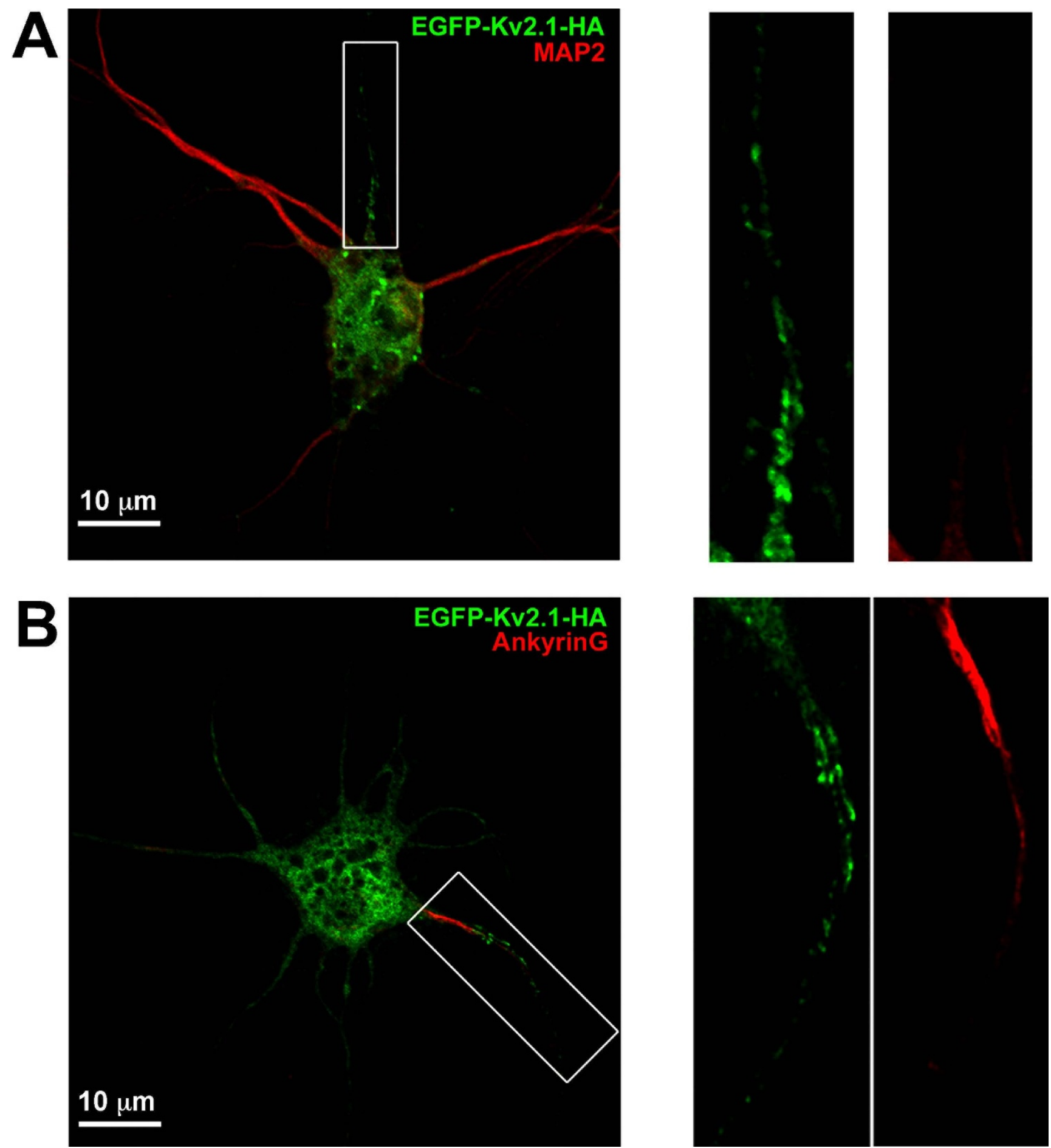

Figure 2

The single proximal neurite containing Kv2.I corresponds to the axon initial segment. Hippocampal neurons grown for 10 days were transfected with EGFP-Kv2.I-HA and fixed $18 \mathrm{~h}$ later. Cultures were then labeled with either antiMAP2 or anti-AnkG followed by Alexa 594-conjugated secondary antibodies. Shown in panel A is an example of Kv2.I clustering in the proximal axon as indicated by the diminished MAP2 staining. Panel B illustrates intense AnkG staining in the Kv2.I positive process. The right hand panels contain enlarged images of the neurites boxed in Panels $A$ and $B$. Note that the diffuse GFP signal in the neurites represents intracellular GFP-Kv2.I. 


\section{Endogenous Kv2.I also preferentially localizes to the AIS} Exogenous expression can lead to artifactual localization. To confirm that Kv2.1 is indeed a component of the AIS in hippocampal cultures, non-transfected cells were immuno-stained for endogenous Kv2.1 and MAP2. Kv2.1 localization in a single proximal neurite with diminished MAP2 staining, i.e. the AIS (marked by the arrow in Fig. 3 ), was observed in 35\% of 7 DIV neurons and $63 \%$ of 10 DIV neurons (200 neurons examined in each of three experiments). The remaining cells showed no AIS localization and a clustered distribution primarily on the cell soma (arrowhead, Fig. 3) as previously reported [2]. Interestingly, as shown in Fig. 3, while all cells showed some Kv2.1 localization to the soma, the most intense staining in DIV 7 and 10 cultures was within the proximal axon. As the cells matured, i.e. in 14 and 21 DIV cultures, the cluster intensity within the cell soma became more equivalent to that in the AIS. We observed Kv2.1 expression on MAP2-positive neurites in only $10 \%$ of the 7 DIV neurons and $2 \%$ of the day 10 and 14 cells. Consistent with reports of multiple axon formation in this culture paradigm [21], 5 and $25 \%$ of the 7 and 14 DIV cells, respectively, showed Kv2.1 accumulation in two AIS domains. By the 14 DIV time point, approximately $87 \%$ of the cells showed dense Kv2.1 expression in one or two AIS domains along with expression over the soma. These expression patterns, and the percentage of cells expressing each pattern, are illustrated in Additional file 1. To further establish that the endogenous Kv2.1 accumulates at the AIS, we used antibodies directed against AnkG in conjunction with endogenous Kv2.1 immuno-detection. Consistent with AIS localization of Kv2.1, the sites of clustering within a proximal neurite corresponded with sites of AnkG enrichment (Additional file 2).

\section{Heterogeneity within the culture system}

We did observe variability in the endogenous Kv2.1 expression pattern, for $12 \%$ of 14 DIV neurons showed no AIS localization. This heterogeneity is not surprising given that hippocampal cultures are heterotypic. Under culture conditions similar to ours, previous reports have placed the amount of pyramidal neurons between 80-90\% whereas glutamic acid decarboxylase (GAD)-positive inhibitory interneurons are present at approximately 10$15 \%$ [2]. Hippocampal cultures were stained with an antiGAD6 antibody in conjunction with anti-Kv2.1 staining to characterize the distribution of Kv2.1 in interneurons as compared to pyramidal neurons. As shown in Fig. 4, the GAD-positive neurons (white arrowhead) did not exhibit AIS localization of Kv2.1 while the GAD-negative neurons did (orange arrows). GAD-positive neurons did display large Kv2.1 clusters mainly within the cell body as previously described [2]. Together, these data suggest Kv2.1 preferentially targets to the proximal region of the pyramidal neuron axons (white arrows). Consistent with previous reports, $10 \%$ of the neurons in our culture were GAD-positive [2].

\section{AIS localized Kv2.I clusters are stable structures containing mobile, but diffusion limited, channels}

Our previous work with the Kv2.1 clusters on the soma shows that while these structures are stable, they are also highly dynamic, often fusing and fragmenting over several min (see Movie S7 from [14]). To address the long term stability of the AIS localized Kv2.1 clusters, we utilized a photoactivatable form of GFP (PAGFP) fused to the N-terminus of Kv2.1 [22]. We co-transfected 7 DIV hippocampal neurons with equal amounts of mRFP-Kv2.1 and PAGFP-Kv2.1-HA and performed live cell image acquisition the following day. Taking advantage of the tetrameric nature of $\mathrm{Kv}$ channels, the mRFP-Kv2.1 construct was included solely to localize the AIS targeted channel for photoactivation. The AIS localized mRFP/PAGFP-Kv2.1 was selected for photoactivation under RFP optics (rectangle, Fig. 5) and successive imaging was performed every two minutes thereafter. As shown in Fig. 5, the photo-activated Kv2.1 within the AIS remained within the activation area and did not significantly re-distribute over the 42 min imaging period. There was no decrease in total AIS associated GFP fluorescence over the imaging period $(8 \times$ $10^{5}$ verses $9.2 \times 10^{5}$ arbitrary units for the Fig. 5 example). These results imply that the Kv2.1 AIS clusters show little lateral diffusion within the membrane and that the channels contained within the AIS have residence times that will be measured in hours as opposed to minutes.

Our previous studies demonstrated that the Kv2.1 surface clusters on the hippocampal cell body are formed not by the tethering of individual channels to sub-membrane scaffolding proteins but rather by the trapping of mobile channels behind a cytoskeletal-based corral $[13,14]$. Given the stable nature of the AIS clusters illustrated in Fig. 5, we next sought to determine whether a similar localization mechanism existed within the AIS. As in our previous work [14], we used FRAP within the cluster to address Kv2.1 mobility within the cluster perimeter. As illustrated in Fig. 6, when one half of an AIS cluster was photo-bleached, GFP fluorescence recovery occurred rapidly with a time constant of $11.1 \mathrm{sec}$ in this representative example. Overall, the mean time constant was $10.4 \pm 4.8$ sec, $n=7$. These time constants are nearly identical to the $11.5 \pm 6.1 \mathrm{sec}$ value observed within clusters on the neuronal cell body [14]. This recovery, along with the corresponding decrease in fluorescence in the unbleached cluster half, is indicative of channel mobility within the cluster perimeter and is most consistent with mobile channels being restrained behind a perimeter fence in the AIS just as they are on the cell body. 

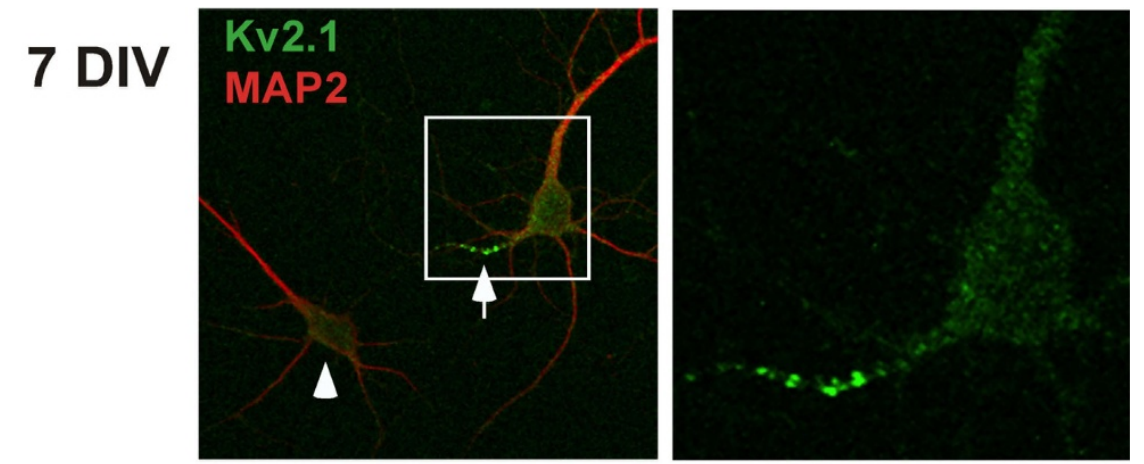

\section{DIV}
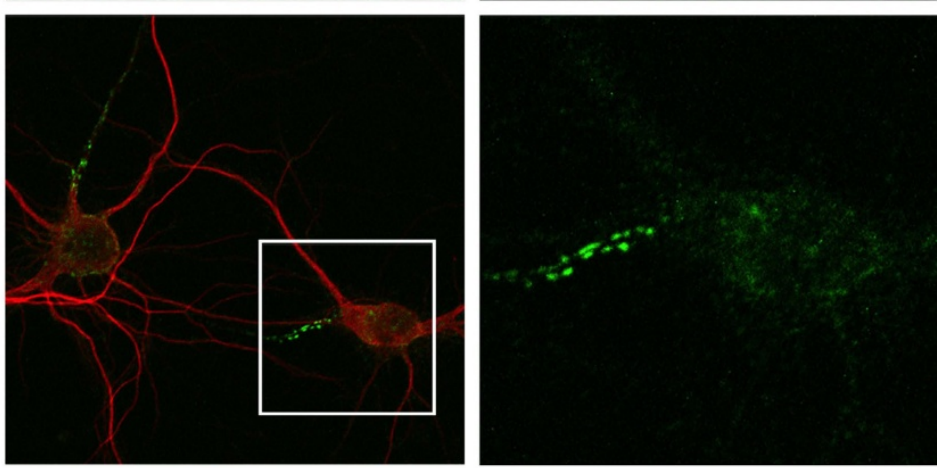

\section{DIV}
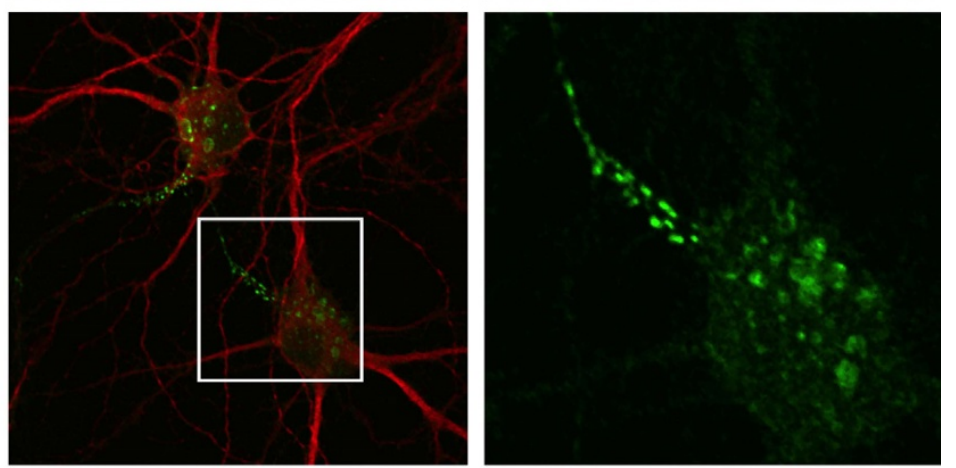

\section{DIV}
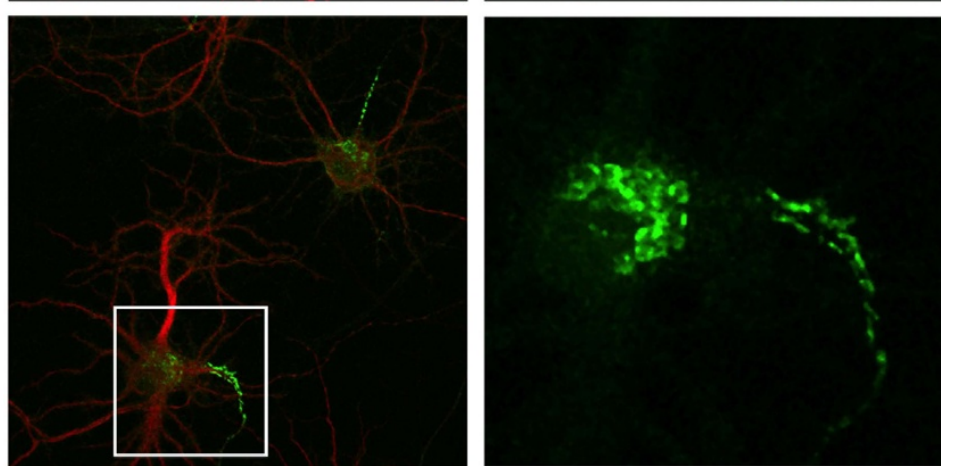

Figure 3

Endogenous Kv2.I preferentially accumulates in a MAP2-negative neurite. Hippocampal cultures grown for 7, 10 , 14 and 2 I days were fixed and immunostained for endogenous Kv2.I (green) and the dendritic marker MAP2 (red). At 7 DIV, the strongest labeling for Kv2.I in these cultures appeared in the proximal portion of a MAP2-negative process (arrow)

although many neurons exhibited faint clusters evenly dispersed over the cell body and proximal neurites (arrowhead). Shown in the right hand panels are maximum projection images corresponding to Kv2.I staining from the boxed regions. In the majority of neurons, the neurite with intense $\mathrm{Kv} 2 . \mathrm{I}$ labeling corresponded to the process with the least intense MAP2 staining, indicating the preferential accumulation of Kv2.I at the proximal axon. 

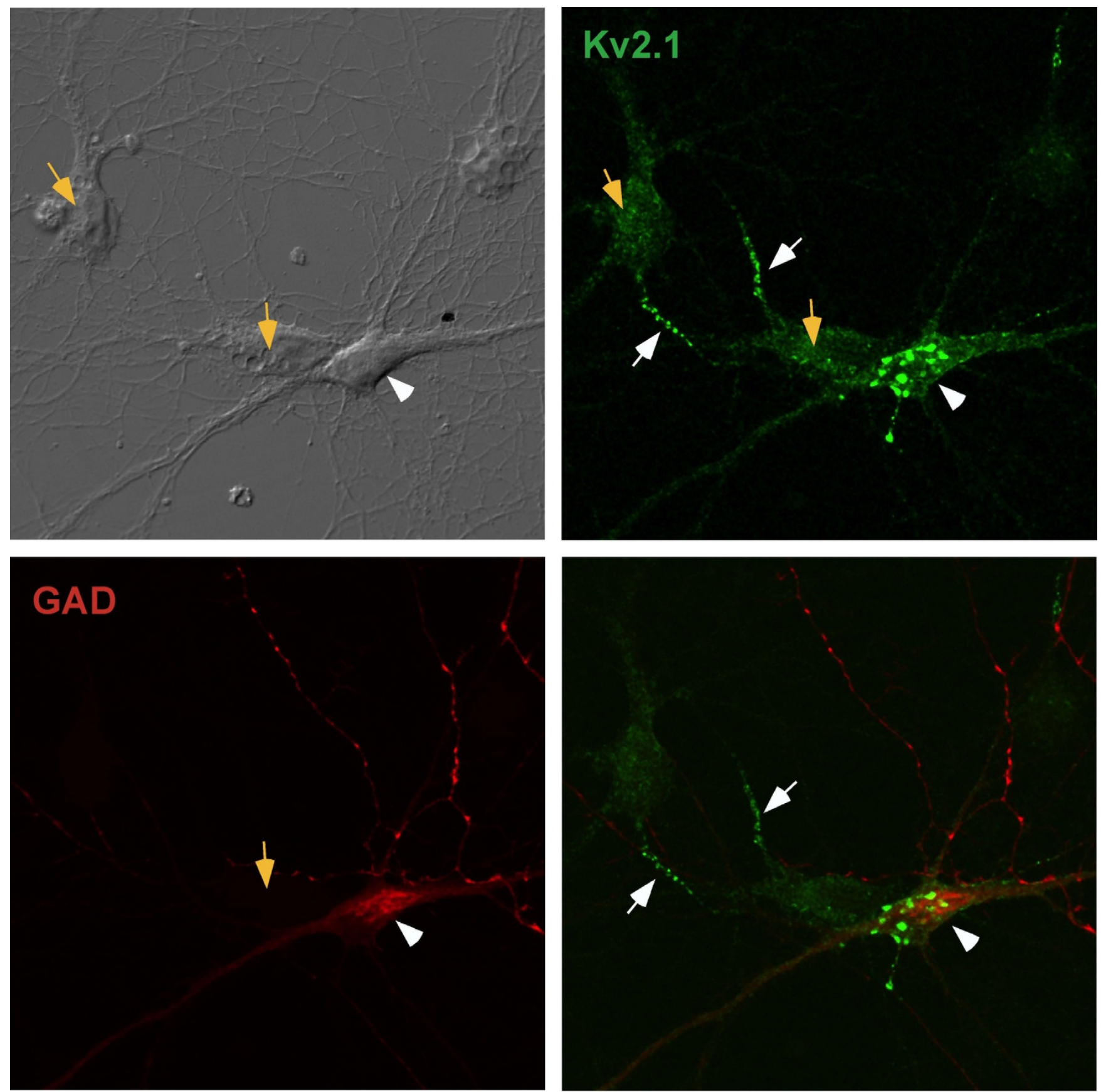

\section{Figure 4}

GAD-positive cells do not exhibit Kv2.I expression in the AIS. DIVI4 hippocampal neurons were fixed and stained for endogenous Kv2.I and glutamic acid decarboxylase (GAD) to identify inhibitory neurons. The orange arrows indicate the GAD-negative neurons while the white arrowhead points to a GAD-positive cell. The white arrows indicate the AIS-localized Kv2.I associated with the GAD-negative neurons. These results are consistent with Kv2.I localization to the AIS only in excitatory, GAD-negative pyramidal neurons.

\section{Kv2. I localizes to hippocampal and cortical AIS domains in} the intact rat brain

The data presented thus far indicate that both the endogenous and exogenously expressed Kv2.1 accumulate at the
AIS in hippocampal cultures, often preferring this region to other cell surface sites early in expression. To demonstrate that the robust AIS localization observed in our culture system is physiologically relevant, we undertook 

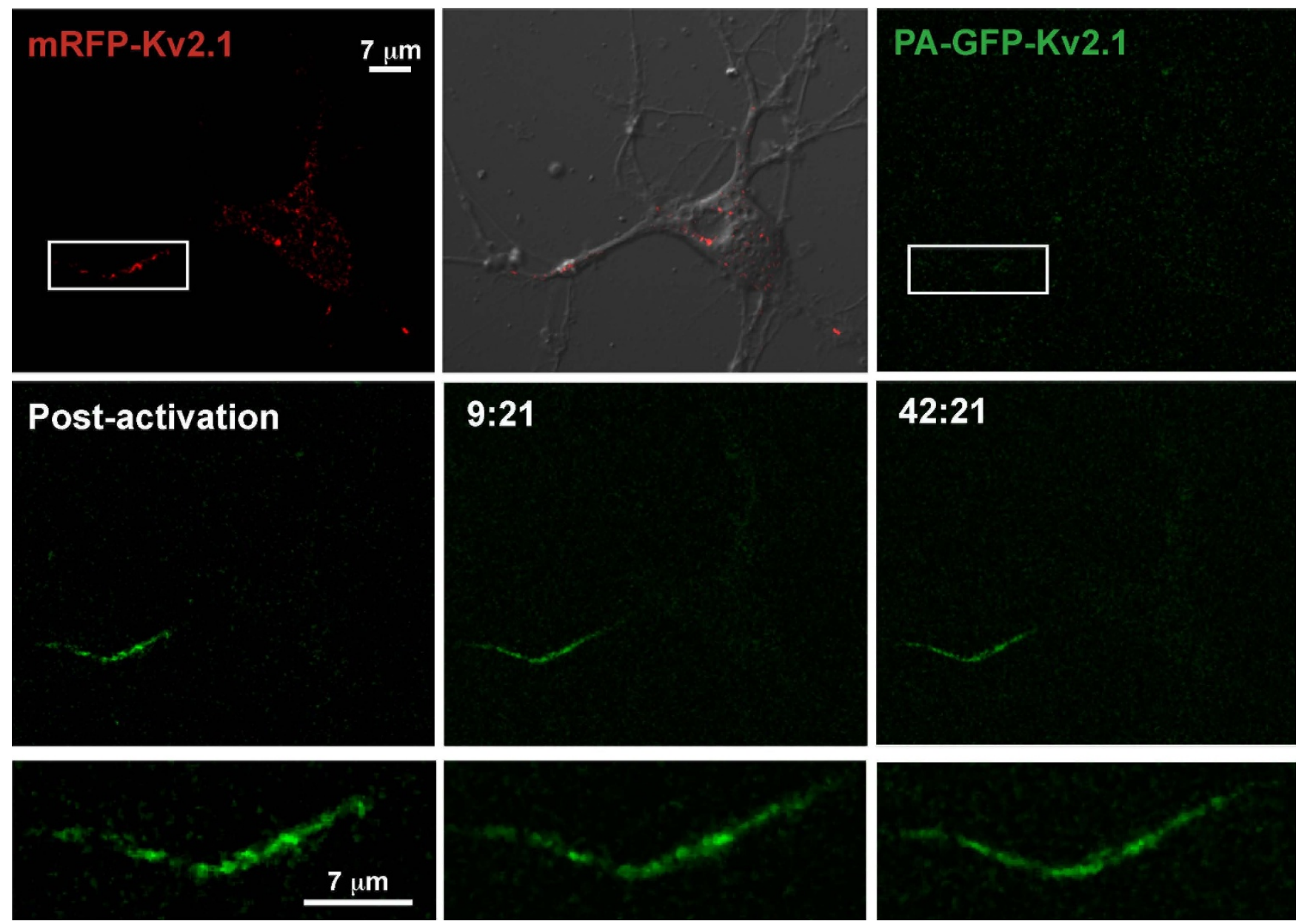

\section{Figure 5}

Kv2.I clusters within the AIS are stable. Shown is a hippocampal neuron co-transfected with a $1: 1$ ratio of mRFP-Kv2.I and PAGFP-Kv2.I-HA plasmid DNA. The proximal region of the single neurite expressing Kv2.I (rectangle) was exposed to $405 \mathrm{~nm}$ laser light to photo-activate the PA-GFP. The top row illustrates the mRFP-Kv2.I signal (red), the mRFP signal overlaid onto the corresponding DIC image and image acquisition under GFP optics. The middle row illustrates the PA-GFP signal immediately after photoactivation and at two representative time points ( $\mathrm{min}: \mathrm{sec}$ ). The bottom row contains enlargements of the activated PA-GFP following photoactivation. Only small changes in cluster size and position occurred over the imaging period. There was no significant decrease in total AIS GFP fluorescence during the 42 minutes of acquisition, suggesting stable retention of Kv2.I within the proximal axon. The imaging interval was 2 min.

immuno-localization studies in rat brain. While Kv2.1 immuno-localization within the rat brain had been previously undertaken by several investigators $[3,8]$, including ourselves (unpublished results), there was no previous suggestion that Kv2.1 exists within the AIS, probably due to the difficulty in identifying the relatively narrow AIS domains within the dense cell body layers of the hippocampus. However, as illustrated in Fig. 7, the use of AnkG staining to identify the AIS (arrows) greatly simplifies matters. Within the hippocampal CA2 layer, Kv2.1 clusters localized over the neuronal cell body as previously described [8] (Fig. 7, panels A-D). However, a linear array of Kv2.1 clusters was also observed within the boundaries of the AnkG positive AIS (arrows), up to $20 \mu \mathrm{m}$ from the cell body (Fig. 7, panel C inset). Consistent with the localization observed in our cultures of hippocampal neurons, not all the ankyrinG-defined AIS domains contained Kv2.1 clusters (arrowhead, panel C). AIS localization was also observed within the CA1 layer (Fig. 7, panel CA1) and CA3 (Fig. 7, panel CA3). Surprisingly, Kv2.1 immuno-localization was not detected in hippocampal sections prior to postnatal day 21 . While channel might be diffusely expressed at these earlier developmental time points, the clustered localization typical of adult tissue was never observed. In adult tissue, the percentage of samples showing Kv2.1 positive AIS domains in the CA1, CA2, and CA3 hippocampal layers were $85(\mathrm{n}=13), 64$ ( $\mathrm{n}$ $=11)$, and $100 \%(\mathrm{n}=17)$, respectively. 

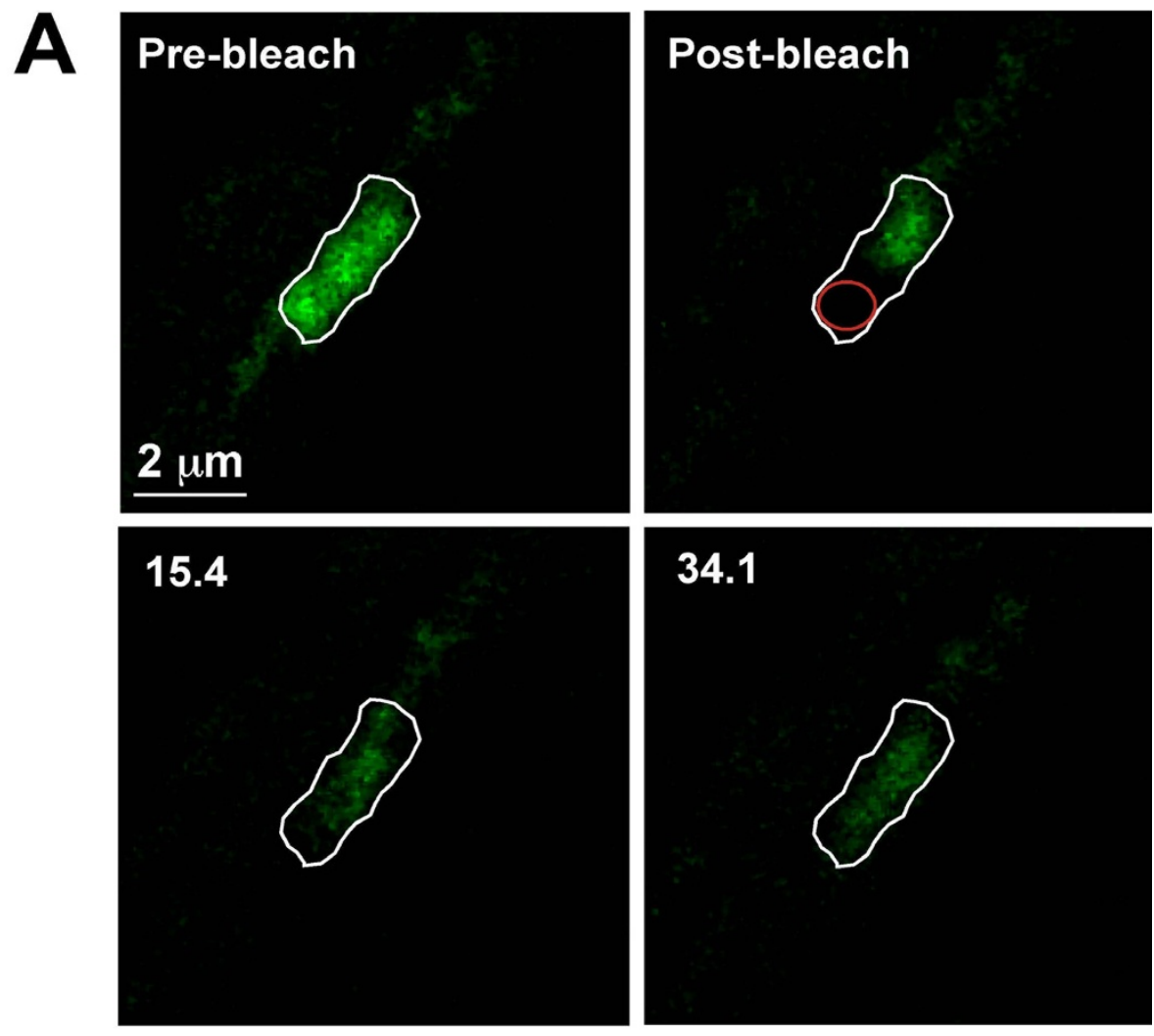

\section{1}

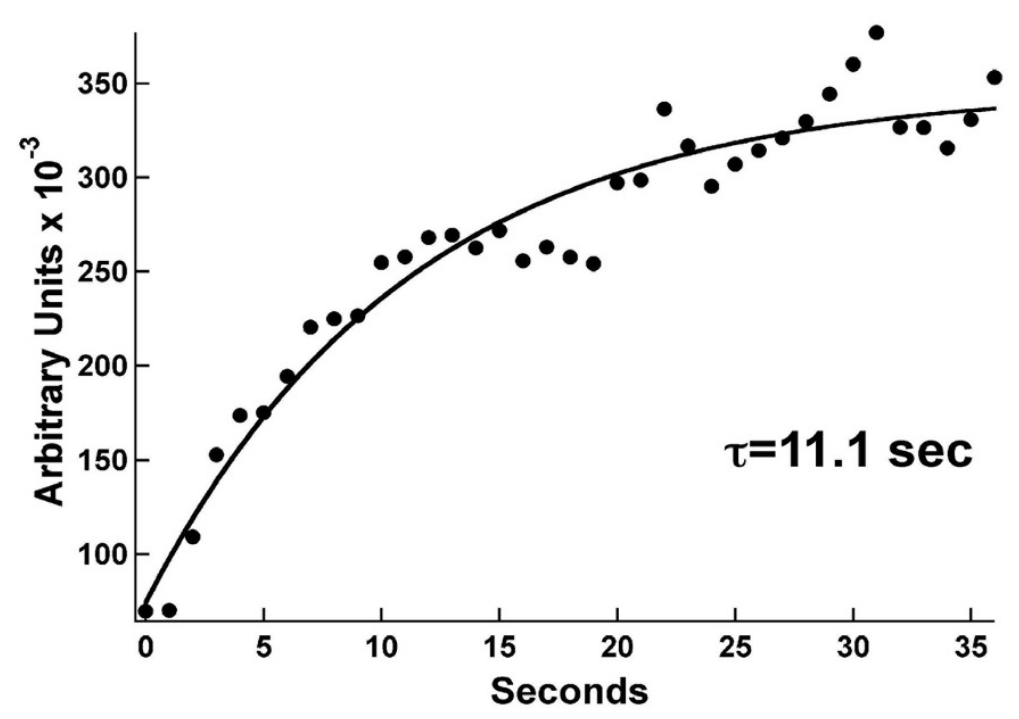

\section{Figure 6}

FRAP analysis indicates Kv2.I channels are mobile within the AIS cluster perimeter. Panel A shows FRAP of an AIS cluster. One half of an AIS cluster (white outline) was photo-bleached and FRAP then monitored every I.I sec. By I5 sec into the recovery, GFP fluorescence was readily observed diffusing from the unbleached half into the bleached region. Panel $B$ shows the FRAP time course. FRAP was quantitated within a small region of interest drawn in the bleached region (red circle). The solid line represents a single exponential fit to the data; $\tau=I I . I$ sec for the cluster illustrated. The mean FRAP time constant for AlS clusters was $10.4 \pm 4.8 \mathrm{sec}, \mathrm{n}=7$. 


\section{CA2}
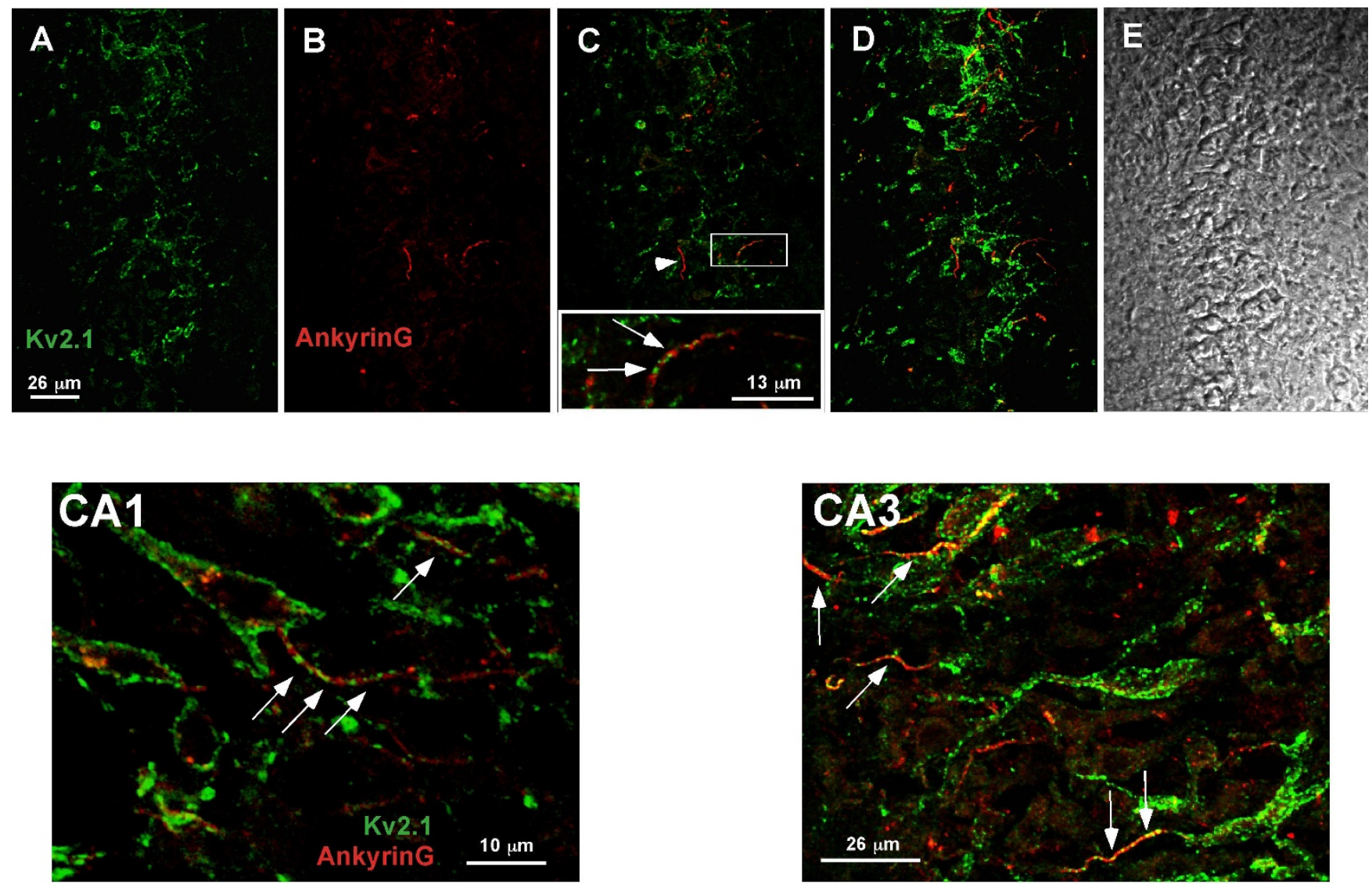

Figure 7

Localization of Kv2.I clusters to the AIS of hippocampal neurons in situ. Postnatal day 21 rat brains were formaldehyde-fixed, cryosectioned and immuno-stained with a polyclonal antibody against Kv2.I and a monoclonal antibody against ankyrin G as described in Methods. The anti-Kv2.I antibody was detected with Alexa 488-conjugated goat anti-rabbit secondary antibody (green) while the anti-AnkG monoclonal antibody was detected with Alexa 594-conjugated goat anti-mouse secondary (red). The top row of panels (A-E) illustrates the localization observed in the CA2 hippocampal layer. Single optical sections of either Kv2.I or ankyrinG immuno-staining are shown in panels $A$ and $B$, respectively. Panel $C$ shows the overlay of these two images with the arrowhead pointing to an AIS domain with little or no Kv2.I immuno-reactivity. However, as the insert containing a magnification of the boxed region indicates, Kv2.I was most often found in the ankyrinG-positive AIS. Panel $D$ shows a maximum projection image (Z-stack compression) and panel $E$ the corresponding DIC image of the same field. The CAI image represents a single optical section within the CAI layer while the CA3 image is a maximum projection image of this hippocampal region. The arrows denote the expression of Kv2.I within the AnkyrinG positive AIS domains.

As shown in Additional file 3, we also observed AIS localization in cultured cortical neurons. Thus, Kv2.1 targeting to the AIS in cortical brain regions was examined also. Fig. 8 illustrates the localization of Kv2.1 to the neuron cell body and AIS in situ in cortical layer IV. In contrast to that observed in the hippocampus, localization of Kv2.1 clusters to the cell body and AIS in cortical sections was observed at early developmental times points, even occasionally at post natal day 2 .

\section{Discussion}

Any Kv2.1 localization to the AIS in cultured hippocampal neurons has been proposed to be a simple tissue culture artifact [8]. However, the data presented above indicate that both transfected and endogenous Kv2.1 often preferentially target to the AIS prior to accumulating at somatic cluster sites. Most importantly, we routinely detected AIS localization of Kv2.1 in the hippocampus and cortex of adult rats, suggesting a role for Kv2.1 in regulating axon excitability in vivo. The AIS-localized Kv2.1 channels reside within clusters similar to those found on 

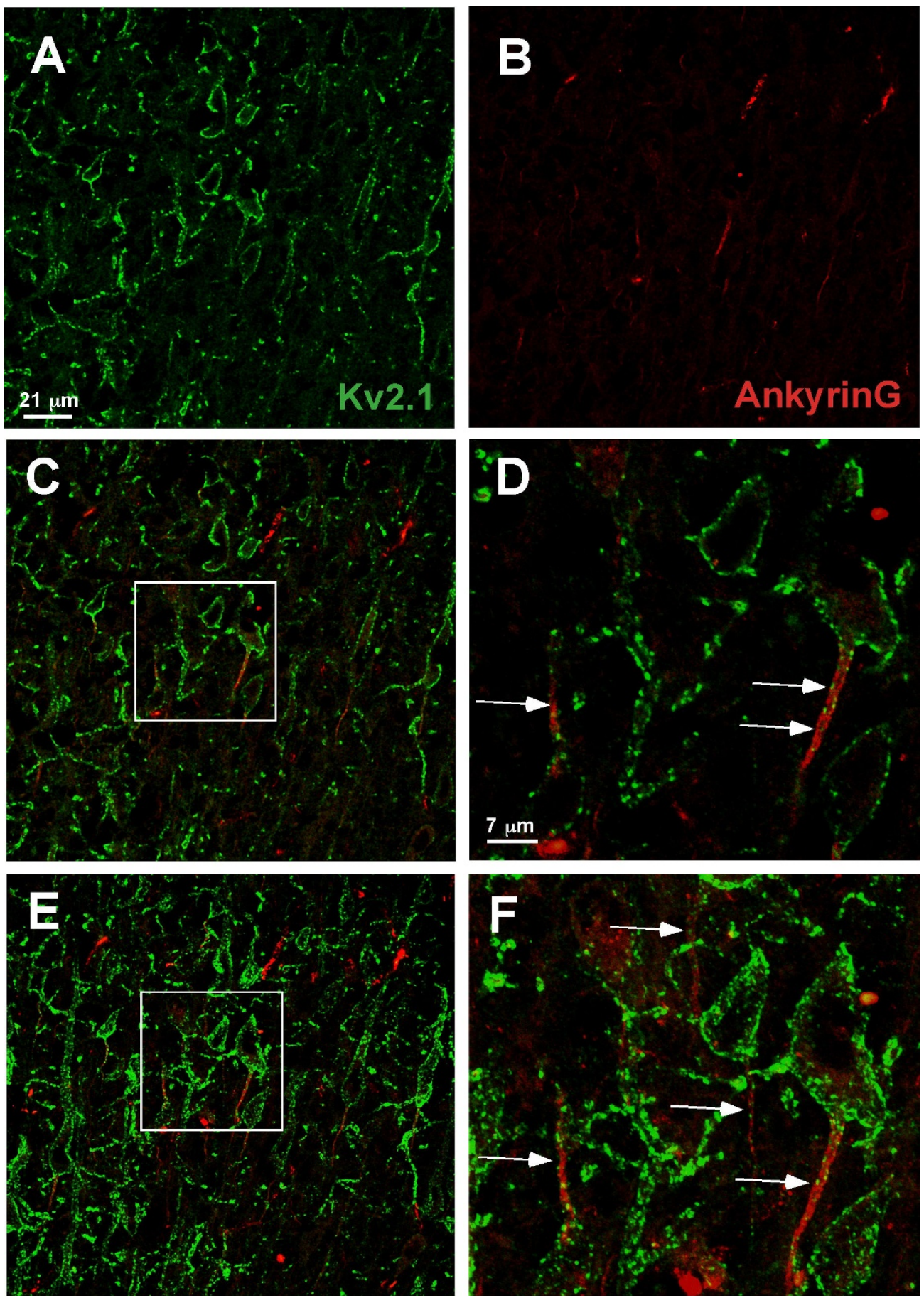

\section{Figure 8}

Localization of Kv2.I clusters to the AIS of layer IV cortical neurons in situ. Rat brain formaldehyde-fixed cyrosections obtained from post natal day $2 \mathrm{I}$ animals were immuno-stained with polyclonal antibody against Kv2.I and monoclonal antibody against ankyrin G. The anti-Kv2.I antibody was detected with Alexa 488-conjugated goat anti-rabbit secondary antibody (green) while the anti-AnkG monoclonal antibody was detected with Alexa 594-conjugated goat anti-mouse secondary antibody (red). Single optical sections of either Kv2.I or ankyrinG immuno-staining are shown in panels $A$ and B. Panel C shows the overlay of these two images and panel $D$ contains a magnification of the boxed region in $C$. Panels $E$ and $F$ show a maximum projection image (Z-stack compression) corresponding to the areas shown in panels $C$ and $D$. The arrows denote the expression of Kv2.I within AnkG-positive AIS domains (arrows). 
the soma in that mobile channels are corralled within a perimeter fence [14]. However, the clusters themselves appear to be more stable within the AIS as compared to their cell body counterparts, consistent with ideas that cytoskeletal structures restrict the diffusion of membrane components within the AIS $[16,17]$.

Our results indicate that transfected Kv2.1 accumulates at the AIS of hippocampal neurons prior to other cell surface sites (Fig. 1). Endogenous channel also concentrates at the AIS in 7 and 10 DIV neurons (Fig. 3). Given that the Kv2.1 surface clusters are sites where trafficking vesicles deliver channel to the cell surface [14], it is possible that vesicles containing newly synthesized Kv2.1 are delivered first to the AIS. A fraction of the AIS localized channel could then be internalized and redistributed over the soma. Another possibility is that Kv2.1 is inserted into the soma membrane and then diffuses into the AIS clusters where it becomes trapped. Such a diffusion-trap mechanism has been proposed for voltage-gated $\mathrm{Na}^{+}$channels that are AIS localized [23]. Alternatively, perhaps delivery occurs equally at the AIS and soma sites but since the AIS is the only neurite receiving the nascent channel, and the newly inserted channel is diluted over all the soma sites, we are left with only the appearance of selective AIS insertion. Future experiments will distinguish between these possibilities.

More than $87 \%$ of 14 DIV neurons demonstrate endogenous Kv2.1 concentration at the AIS. Why this localization has not been observed by other investigators is unclear. Perhaps subtle differences in tissue culture conditions could account for the discrepancy. Previous Kv2.1 localizations in adult brain, as performed by other investigators $[3,8]$ and ourselves (unpublished results), also failed to detect Kv2.1 expression in the AIS. It is possible that the AIS localization was missed because without simultaneous ankyrinG staining and high resolution confocal imaging, it is difficult to identify the AIS structure within the dense layers of neuronal cell bodies in the hippocampus. The finding that not all hippocampal neurons show Kv2.1 localization in the AIS agrees with the Fig. 4 data showing a lack of AIS localization in GAD-positive inhibitory neurons.

\section{Conclusion}

While a high concentration of sodium channels at the AIS ensures axonal action potential generation [24], it is not clear what role Kv2.1 plays in this specialized cell surface domain. Kv2.1 has slow activation kinetics and is therefore unlikely to contribute significantly to repolarization during a single action potential. However, Kv2.1 could regulate axonal excitability following trains of action potentials, for studies by McBain and co-workers indicate that reduction of Kv2.1 in pyramidal neurons results in a broadening of the action potential waveform [5]. Recently, the Kv2.2 channel, which cannot form heterotetrameric complexes with Kv2.1, has been localized to the AIS of neurons in the medial nucleus of the trapezoid body [25]. Kv2.2, which is functionally similar to Kv2.1, is proposed to enhance the recovery of AIS sodium channels from the inactive state by hyperpolarizing the membrane potential during repetitive action potential firing [25]. Kv2.1 could be performing a similar function. In addition, concentration of Kv2.1 at the AIS could regulate the back-propagation of depolarization into the soma. Given that multiple phosphorylation sites within the Kv2.1 C-terminus influence the voltage-dependence of activation [26], the phosphorylation of AIS-localized Kv2.1 provides a mechanism to regulate axonal excitability.

\section{Methods \\ Primary hippocampal cell cultures}

Neurons from embryonic day 18 rat pups were cultured as previously described [27]. Briefly, neurons from cryo-preserved E1 8 rat hippocampal dissociations were plated at a density of $\sim 15,000-30,000$ cells/cm2 on poly-D-lysine coated glass-bottom dishes (MatTek) in glial-cell conditioned neurobasal medium (GCM) containing B27 supplement (Invitrogen) [28]. Every 3-4 days after plating, one-half of the culture medium was replaced with GCM. Neurons plated on $35 \mathrm{~mm}$ glass bottom dishes and cultured for 7-10 days were transfected with $2.0 \mu$ of Lipofectamine 2000 and $0.75 \mu \mathrm{g}$ of Kv2.1-expressing plasmid DNA in $100 \mu$ l OptiMem (Invitrogen). Two-hours after transfection, the culture medium was replaced with fresh Neurobasal/B27. The expression plasmid constructs containing fluorescent protein and epitope-tagged Kv2.1 have been previously described $[13,14,22,29]$. In brief, EGFP was placed onto the Kv2.1 N-terminus and two HA epitopes were inserted in the extracellular loop between S1 and S2 transmembrane domains.

\section{Live cell imaging}

All image acquisition was performed using an Olympus Fluoview 1000 confocal microscope system as previously described $[13,14]$. For live cell acquisition, the objective and microscope stage were maintained at $37^{\circ} \mathrm{C}$. Prior to imaging, media was replaced with pre-warmed Hepesbuffered $(25 \mathrm{mM})$ Neurobasal medium containing B-27 supplement (Invitrogen). GFP was excited using the 488 $\mathrm{nm}$ line of an Ar laser set at $0.1-0.5 \%$ transmission and emission collected using the variable bandpass filter set at 500-550 nm. A 60×, 1.4 NA oil immersion objective was used for imaging and the pinhole diameter set for 1 Airy Unit. For each image, the detector voltage was adjusted as necessary to utilize the full 12-bit linear range. For the imaging of individual Kv2.1-containing clusters, an optical zoom of 8-10 $\times$ was often used. Images were acquired 
every $0.5-120$ seconds as indicated at either $512 \times 512$ or $1024 \times 1024$ resolution. Cells were imaged for less than one hour on the microscope stage.

For FRAP analysis of GFP-Kv2.1 within the cluster perimeter, a circular region of interest (ROI) containing approximately half of the cluster area was photobleached using the SIM scanner of the Olympus FV1000 in tornado scan mode with a $405 \mathrm{~nm}$ diode laser at $12-20 \%$ transmission for $0.5-1$ seconds as previously described $[13,14]$. The SIM scanner was synchronized with the main scanner during bleach and acquisition. Following bleach, imaging was performed by raster scanning with the $488 \mathrm{~nm}$ line of a $40 \mathrm{~mW}$ Ar laser at $0.2-0.5 \%$ transmission. The variable bandpass filter was set to detect $505-530 \mathrm{~nm}$ emission and images were acquired every $0.5-1.1$ seconds at $512 \times$ 512 resolution. Fluorescence intensity, in arbitrary units, within the bleach ROI was fit according to Eq. 1 as previously described $[13,22]$.

$$
f(x)=\sum_{i=1}^{n} A_{i}\left(1-e^{\left(-t / \tau_{i}\right)}\right)
$$

where $A_{i}$ is the amplitude of each component, $t$ is time and $\tau_{\mathrm{i}}$ is the time constant of each component.

\section{Immunofluorescence}

Detection of surface GFP-Kv2.1-HA was performed by incubating live cells with a 1:2000 dilution of anti-HA Alexa-594 conjugated monoclonal antibody (Molecular Probes) in Hepes-buffered Neurobasal/B27 for a minimum of $30 \mathrm{~min}$ at $37^{\circ} \mathrm{C}$. Only live cells were used for surface channel detection since fixation permits labeling of internal, non-surface channel (data not shown). For endogenous Kv2.1, ankyrinG and MAP2 immuno-staining, neurons were fixed in 4\% formaldehyde, $4 \%$ sucrose in PBS for 15 min, incubated in 0.5\% CHAPS, blocked in $5 \%$ non-fat milk and $1 \%$ goat serum in PBS, and labeled with the indicated antibody diluted in 1\% BSA in PBS. Antibody and dilutions were as follows: affinity-purified rabbit anti-Kv2.1 polyclonal antibody (Upstate Biotechnology, 1:200 dilution, raised against amino acids 837853); mouse monoclonal anti-MAP-2 (Sigma, 1:2000); mouse monoclonal anti-AnkyrinG (Zymed, 1:100, raised against the spectrin binding domain); and mouse monoclonal anti- $\alpha$-glutamic acid decarboxylase (Developmental Studies Hybidoma Bank, 1:50). Goat anti-mouse and goat anti-rabbit secondary antibodies conjugated to Alexa 488 or 594 (Molecular Probes) were diluted 1:1000 in 1\% BSA/PBS.

Hippocampal slices were prepared from post natal day 21 and adult rats. In accordance with university guidelines, the animals were anesthetized with ketamine and decapitated. After decapitation, the brains were removed and fixed with 4\% paraformaldehyde (PFA) for 4 hours at $4^{\circ} \mathrm{C}$, replacing the PFA solution after 2 hours. The tissue was cryoprotected in $30 \%$ sucrose for 2 days at $4^{\circ} \mathrm{C}$. Following cryoprotection and freezing, sagittal sections (16 $\mu \mathrm{m})$ were prepared on a cryostat microtome. In order to diminish non-specific binding, the tissue sections were incubated overnight in $1 \times \mathrm{PBS} / 5 \%$ dry milk/10\% goat serum at $4{ }^{\circ} \mathrm{C}$. The detection of endogenous Kv2.1 and ankyrinG was performed by incubating the tissue sections with affinity-purified anti-Kv2.1 polyclonal (1:500, Upstate Biotechnology) and anti-Ankyrin G monoclonal (1:500, Zymed) antibodies diluted in $1 \times \mathrm{PBS} / 10 \%$ goat serum $/ 0.5 \% \mathrm{CHAPS}$ overnight at $4^{\circ} \mathrm{C}$. Following primary antibody incubation, brain slices were washed three times with $1 \times \mathrm{PBS} / 0.5 \% \mathrm{CHAPS}$. The tissue slices were incubated with Alexa 488-conjugated goat anti-rabbit secondary (1:500, Invitrogen) and Alexa 594-conjugated goat anti-mouse secondary (1:500, Molecular Probes) for 1 hour at room temperature. Following secondary antibody incubation, the brain slices were washed three times with $1 \times$ PBS/0.5\% CHAPS and mounted using AquaPolymount (Polysciences).

\section{Specificity of antibody binding}

The detection of MAP2, ankyrinG, GAD and Kv2.1 in cultured hippocampal neurons has been performed by multiple groups using either the same antibodies as used here or closely related ones $[2,7,18,20,30-36]$. Importantly, the immunolocalization patterns for endogenous Kv2.1 in the hippocampal cultures are identical to the patterns observed with GFP-Kv2.1-HA under GFP optics or with anti-HA immuno-staining. With respect to the anti-HA antibody specificity, cell surface staining was never observed in GFP-Kv2.1-HA free neurons.

An additional control is required for the Kv2.1 localization to the ankyrinG defined AIS in brain tissue sections, as this has not been reported previously. Since immunogen block controls are known to be misleading [37], we also performed immuno-localization of ankyring and Kv2.1 using a monoclonal antibody for Kv2.1 and a polyclonal antibody for ankyrinG. A mouse monoclonal against Kv2.1 (1:500 dilution, Upstate Biotechnology, raised against a GST fusion protein containing amino acids 509-853) was used in conjunction with a rabbit anti-ankyrinG polyclonal (1:500 dilution, Santa Cruz Biotechnology, raised against amino acids 4163-4377). While the immuno-staining was not as robust as that observed with the other antibody combination, the same AIS staining patterns for ankyring and Kv2.1 were observed as shown in Additional file 4. It is highly unlikely that these distinct anti-Kv2.1 antibodies both nonspecifically bind AIS structures, especially since GFPKv2.1-HA also targets to this region in transfected neurons. 


\section{Image analysis}

Offline image analysis was done using the Olympus FV1000 software (version 1.03) and Volocity 4.4 (Improvision, Lexington, MA). Data analysis and curve-fitting was performed with SigmaPlot 8 (Systat, Point Richmond, CA) or IgorPro 5.03 (Wavemetrics, Portland, OR). Images were filtered in Volocity using a $3 \times 3$ median filter. Compilation of images was performed using Adobe Photoshop and contrast and brightness adjustments were made. Both compressed (maximum projection) and single Z-section images are displayed as indicated.

\section{Abbreviations}

AIS: Axon initial segement; AnkG: Ankyrin G; DIV: Days in vitro; GAD: glutamic acid decarboxylase.

\section{Authors' contributions}

PDS and MMT designed the experimental procedures, prepared in vitro cultures, and performed imaging and analysis. CMW performed tissue sectioning and immunostaining. The article was written by PDS and MMT.

\section{Additional material}

\section{Additional file 1}

Summary of the endogenous Kv2.1 localization patterns in cultured hippocampal neurons. The bar graph of panel A illustrates the percentages of neurons in 7, 10, and 14 DIV cultures that showed the expression patterns illustrated in B. Neurons were formaldehyde-fixed and immunostained with polyclonal antibody against Kv2.1 and monoclonal antibody against MAP2. The anti-Kv2.1 antibody was detected with Alexa 488conjugated goat anti-rabbit secondary antibody (green) while the antiMAP2 monoclonal antibody was detected with Alexa 594-conjugated goat anti-mouse secondary antibody (red). The images represent a maximum projection image. The arrows denote the expression of Kv2.1 within a proximal neurite.

Click here for file

[http://www.biomedcentral.com/content/supplementary/14712202-9-112-S1.jpeg]

\section{Additional file 2}

Endogenous Kv2.1 localizes to the axon initial segment as defined by AnkyrinG enrichment. Hippocampal neurons grown for 14 DIV were fixed and immuno-stained for the AIS marker Ankyrin G and endogenous Kv2.1. Shown are superimposed images of neurons stained for Kv2.1 (green) and AnkyrinG (red) from single basal z-sections. Individual fluorescent images, corresponding to boxed regions in the overlay, are shown to the right.

Click here for file

[http://www.biomedcentral.com/content/supplementary/14712202-9-112-S2.jpeg]

\section{Additional file 3}

Localization of endogenous Kv2.1 to the AIS of cultured cortical neurons. Cortical neurons were isolated from E1 8 rat cortex by mechanical dissociation following treatment with $0.25 \%$ trypsin. Cells were grown on poly-lysine coated glass bottomed dishes in Neurobasal/B27/PenStrep for 10 days were formaldehyde-fixed and immuno-stained with polyclonal antibody against Kv2.1 and monoclonal antibody against MAP2. The anti-Kv2.1 antibody was detected with Alexa 488-conjugated goat antirabbit secondary antibody (green) while the anti-MAP2 monoclonal antibody was detected with Alexa 594 conjugated goat anti-mouse secondary antibody (red). The image represents a single optical section at the level of the substrate-attached neurites. The arrows denote the expression of Kv2.1 within a MAP2 negative neurite that is defined as the AIS.

Click here for file

[http://www.biomedcentral.com/content/supplementary/14712202-9-112-S3.jpeg]

\section{Additional file 4}

Localization of Kv2.1 to the AIS in the CA1 layer using a monoclonal anti-Kv2.1 antibody paired with a polyclonal anti-ankyrinG antibody. Postnatal day 21 rat brains were formaldehyde-fixed, cryosectioned and immuno-stained with a monoclonal antibody against Kv2.1 and polyclonal clonal antibody against ankyrin $G$ as described in Methods. The antiKv2.1 antibody was detected with Alexa 594-conjugated goat anti-mouse secondary antibody (red) while the anti-ankyrin $G$ monoclonal antibody was detected with Alexa 488-conjugated goat anti-rabbit secondary (green). The image represents a single optical section. The arrows denote the expression of Kv2.1 within the AnkG positive AIS domain.

Click here for file

[http://www.biomedcentral.com/content/supplementary/14712202-9-112-S4.jpeg]

\section{Acknowledgements}

This research was supported by NIH grants NS4I542 and HL49330 to MMT and training grant NS43 I 15 to PDS. The anti-GAD was obtained from the Developmental Studies Hybridoma Bank developed under the auspices of the NICHD and maintained by The University of lowa, Department of Biological Sciences, lowa City, IA 52242. The authors thank Dr. Noreen Reist for comments on the manuscript.

\section{References}

I. Scannevin RH, Murakoshi H, Rhodes KJ, Trimmer JS: Identification of a cytoplasmic domain important in the polarized expression and clustering of the Kv2.I K+ channel. J Cell Biol 1996, 135:1619-1632.

2. Antonucci DE, Lim ST, Vassanelli S, Trimmer JS: Dynamic localization and clustering of dendritic Kv2.I voltage-dependent potassium channels in developing hippocampal neurons. Neuroscience 200I, 108:69-8I.

3. Du J, Tao-Cheng JH, Zerfas P, McBain CJ: The K+ channel, Kv2.I, is apposed to astrocytic processes and is associated with inhibitory postsynaptic membranes in hippocampal and cortical principal neurons and inhibitory interneurons. Neuroscience 1998, 84:37-48.

4. Muennich EA, Fyffe RE: Focal aggregation of voltage-gated, Kv2.I subunit-containing, potassium channels at synaptic sites in rat spinal motoneurones. J Physiol 2004, 554:673-685.

5. Du J, Haak LL, Phillips-Tansey E, Russell JT, McBain CJ: Frequencydependent regulation of rat hippocampal somato-dendritic excitability by the $\mathrm{K}+$ channel subunit Kv2.I. J Physiol 2000, 522(Pt I): |9-3|.

6. Misonou H, Trimmer JS: Determinants of voltage-gated potassium channel surface expression and localization in Mammalian neurons. Crit Rev Biochem Mol Biol 2004, 39: I25-I45. 
7. Misonou H, Mohapatra DP, Park EW, Leung V, Zhen D, Misonou K, et al.: Regulation of ion channel localization and phosphorylation by neuronal activity. Nat Neurosci 2004, 7:71 I-7I8.

8. Misonou H, Mohapatra DP, Menegola M, Trimmer JS: Calcium- and metabolic state-dependent modulation of the voltagedependent Kv2.I channel regulates neuronal excitability in response to ischemia. I Neurosci 2005, 25: I I I84- I I I 93.

9. Mohapatra DP, Trimmer JS: The Kv2.I C terminus can autonomously transfer Kv2. I-like phosphorylation-dependent localization, voltage-dependent gating, and muscarinic modulation to diverse Kv channels. J Neurosci 2006, 26:685-695.

10. Misonou H, Mohapatra DP, Trimmer JS: Kv2.I: A Voltage-Gated K(+) Channel Critical to Dynamic Control of Neuronal Excitability. Neurotoxicology 2005.

II. Pal S, Hartnett KA, Nerbonne JM, Levitan ES, Aizenman E: Mediation of neuronal apoptosis by Kv2. I-encoded potassium channels. J Neurosci 2003, 23:4798-4802.

12. Pal SK, Takimoto K, Aizenman E, Levitan ES: Apoptotic surface delivery of K+ channels. Cell Death Differ 2006, I 3:66 I-667.

13. Tamkun MM, O'Connell KM, Rolig AS: A cytoskeletal-based perimeter fence selectively corrals a sub-population of cell surface Kv2. I channels. J Cell Sci 2007, I 20:24I 3-2423.

14. O'Connell KM, Rolig AS, Whitesell JD, Tamkun MM: Kv2. I potassium channels are retained within dynamic cell surface microdomains that are defined by a perimeter fence. J Neurosci 2006, 26:9609-9618.

I5. Kusumi A, Nakada C, Ritchie K, Murase K, Suzuki K, Murakoshi H, et al.: Paradigm shift of the plasma membrane concept from the two-dimensional continuum fluid to the partitioned fluid: high-speed single-molecule tracking of membrane molecules. Annu Rev Biophys Biomol Struct 2005, 34:35I-378.

16. Nakada C, Ritchie K, Oba Y, Nakamura M, Hotta Y, lino R, et al: Accumulation of anchored proteins forms membrane diffusion barriers during neuronal polarization. Nat Cell Biol 2003 Jul; 5(7): 626-32 2003, 5:626-632.

17. Winckler B, Forscher P, Mellman I: A diffusion barrier maintains distribution of membrane proteins in polarized neurons. Nature 1999, 397:698-701.

18. Lim ST, Antonucci DE, Scannevin RH, Trimmer JS: A novel targeting signal for proximal clustering of the Kv2. I K+ channel in hippocampal neurons. Neuron 2000, 25:385-397.

19. Boiko T, Vakulenko M, Ewers H, Yap CC, Norden C, Winckler B: Ankyrin-dependent and -independent mechanisms orchestrate axonal compartmentalization of LI family members neurofascin and LI/neuron-glia cell adhesion molecule. J Neurosci 2007, 27:590-603.

20. Garrido J], Giraud P, Carlier E, Fernandes F, Moussif A, Fache MP, et al.: A targeting motif involved in sodium channel clustering at the axonal initial segment. Science 2003, 300:2091-2094.

21. Yoshimura T, Kawano Y, Arimura N, Kawabata S, Kikuchi A, Kaibuchi K: GSK-3beta regulates phosphorylation of CRMP-2 and neuronal polarity. Cell 2005, I 20(I): I37-I49.

22. O'Connell KM, Tamkun MM: Targeting of voltage-gated potassium channel isoforms to distinct cell surface microdomains. J Cell Sci 2005, I I 8:2 I 55-2 I 66

23. Fache MP, Moussif A, Fernandes F, Giraud P, Garrido IJ, Dargent B: Endocytotic elimination and domain-selective tethering constitute a potential mechanism of protein segregation at the axonal initial segment. Journal of Cell Biology 2004, I 66:57|-578.

24. Colbert CM, Pan E: Ion channel properties underlying axonal action potential initiation in pyramidal neurons. Nat Neurosci 2002 Jun; 5(6): 533-8 2002, 5:533-538.

25. Johnston J, Griffin SJ, Baker C, Skrzypiec A, Chernova T, Forsythe ID: Initial segment Kv2.2 channels mediate a slow delayed rectifier and maintain high frequency action potential firing in MNTB neurones. J Physiol 2008.

26. Park KS, Mohapatra DP, Misonou H, Trimmer JS: Graded regulation of the Kv2. I potassium channel by variable phosphorylation. Science 2006, 3 I 3:976-979.

27. Minamide LS, Striegl AM, Boyle JA, Meberg PJ, Bamburg JR: Neurodegenerative stimuli induce persistent ADF/cofilin-actin rods that disrupt distal neurite function. Nat Cell Biol 2000 , 2:628-636.

28. Meberg PJ, Miller MW: Culturing hippocampal and cortical neurons. Neurons: Methods and Applications for the Cell Biologist 2003, 7I:III-I27.
29. O'Connell KM, Whitesell JD, Tamkun MM: Localization and mobility of the delayed-rectifer $\mathrm{K}+$ channel $\mathrm{Kv2}$.I in adult cardiomyocytes. Am J Physiol Heart Circ Physiol 2008, 294:H229-H237.

30. Garrido JJ, Fernandes F, Moussif A, Fache MP, Giraud P, Dargent W: Dynamic compartmentalization of the voltage-gated sodium channels in axons. Biology of the Cell 2003, 95:437-445.

31. Hedstrom KL, Xu X, Ogawa Y, Frischknecht R, Seidenbecher $\mathrm{Cl}$, Shrager $P$, et al: Neurofascin assembles a specialized extracellular matrix at the axon initial segment. J Cell Biol 2007, I 78:875-886.

32. LeMaillet G, Walker B, Lambert S: Identification of a conserved ankyrin-binding motif in the family of sodium channel alpha subunits. J Biol Chem 2003, 278:27333-27339.

33. Pan Z, Kao T, Horvath Z, Lemos J, Sul JY, Cranstoun SD, et al: A common ankyrin-G-based mechanism retains $K C N Q$ and $\mathrm{NaV}$ channels at electrically active domains of the axon. Neurosci 2006, 26:2599-26I3.

34. Rasmussen HB, Frokjaer-Jensen C, Jensen CS, Jensen HS, Jorgensen NK, Misonou $\mathrm{H}$, et al.: Requirement of subunit co-assembly and ankyrin-G for M-channel localization at the axon initial segment. J Cell Sci 2007, I 20:953-963.

35. Van Wart A, Trimmer JS, Matthews G: Polarized distribution of ion channels within microdomains of the axon initial segment. J Comp Neurol 2007, 500:339-352.

36. Yang $Y$, Ogawa $Y$, Hedstrom KL, Rasband $M N$ : betalV spectrin is recruited to axon initial segments and nodes of Ranvier by ankyrinG. J Cell Biol 2007, I 76:509-5I9.

37. Saper CB, Sawchenko PE: Magic peptides, magic antibodies: guidelines for appropriate controls for immunohistochemistry. J Comp Neurol 2003, 465:161-163.
Publish with Biomed Central and every scientist can read your work free of charge

"BioMed Central will be the most significant development for disseminating the results of biomedical research in our lifetime. "

Sir Paul Nurse, Cancer Research UK

Your research papers will be:

- available free of charge to the entire biomedical community

- peer reviewed and published immediately upon acceptance

- cited in PubMed and archived on PubMed Central

- yours - you keep the copyright
BiolMedcentral 